\title{
Perspectives on Health and Usage Monitoring Systems (HUMS) of helicopters
}

\author{
Alexandre Mauricio ${ }^{1,2}$, Junyu $\mathrm{Qi}^{1,2}$, Linghao Zhou ${ }^{3}$, Wenyi $\mathrm{Wang}^{4}$, David $\mathrm{Mba}^{5}$, \\ and Konstantinos Gryllias 1,2,* \\ ${ }^{1}$ Department of Mechanical Engineering, KU Leuven, 3001, Leuven, Belgium \\ ${ }^{2}$ Dynamics of Mechanical and Mechatronic Systems, Flanders Make, 3001, Leuven, Belgium \\ ${ }^{3}$ London South Bank University, School of Engineering, SE1 0AA, London, UK \\ ${ }^{4}$ Defence Science and Technology Organization (DSTO), Air Vehicles Division, Melbourne, VIC \\ 3011, Australia \\ ${ }^{5}$ De Montfort University, Faculty of Technology, LE1 9BH, Leicester, UK
}

\begin{abstract}
Helicopters are extensively used in civil applications as they are versatile in their capabilities to manoeuvre. Their operation under harsh conditions and environments demand for a strict maintenance plan. Main gearboxes (MGB) of helicopters are a critical component responsible for reducing the high input speed generated from the gas turbine engines. Health and Usage Monitoring Systems (HUMS) are installed in an effort to monitor the health state of the transmission systems, and ideally, to detect and diagnose the type of a generated fault. Even though the development of HUMS contributed to the reduction of worldwide helicopter accident rate, more advanced systems are needed based on the investigation of the air accidents of AS332 L2 Super Puma in Scotland in 2009 and of EC225 LP Super Puma in Bergen in 2016, due to failure of a planet gear of the MGB. A plethora of signal processing methodologies have been proposed for the early detection of faults but often they fail in complex structures, such as planetary gearboxes operating under various conditions. In this paper the performance of a recently proposed diagnostic tool, called IESFOgram, is evaluated and compared with state of the art techniques, applied on signal captured on a Category A Super Puma SA330 MGB.
\end{abstract}

\section{Introduction}

Health and Usage Monitoring Systems (HUMS) conduct helicopter health monitoring by extracting Health Indicators (HI) from acquired vibration data. However, HI's have a wide range of values depending on a plethora of conditions of the helicopter besides their health state (e.g. temperature, speed, torque, etc.), making the implementation of specific thresholds of the HI often unreliable for the health monitoring. Analysis of the time-domain of the vibration data is also non-practical, as MGB of helicopters are complex machinery with many sources. Analysis of the frequency-domain provides a more direct way to monitor the health condition of its components by tracking the amplitude at specific frequencies related to specific components. Signal processing techniques are usually applied in this scenario in

\footnotetext{
* Corresponding author: konstantinos.gyllias@kuleuven.be
} 
order to detect modulations related to the fault that may be hidden in the spectrum $[1,2]$. One of the most common approaches in bearing fault detection is the Envelope Analysis, where the signal is demodulated in order to detect the lower cyclic modulations of the bearing. This method is sensitive to the noise, so it is commonly used alongside with a band pass filter around the band of frequencies excited by the impulses of the bearing fault. The selection of the band pass filter can be made mainly by two general approaches: exploitation of engineering knowledge and experience; or through band selection tools. The band selection tools provide the advantage of (semi-) automated selection without requiring expertise and/or trial-and-error of band-pass filtering. The most widely used method is the Fast Kurtogram, which maximises the value of Spectral Kurtosis of a series of filters. However, there are other known phenomena that generate high Kurtosis values, resulting in the selection of incorrect bands [3]. In the frames of the analysis of vibration data of a MGB with damaged bearing, Zhou et. al. [1] concluded that the Kurtogram was ineffective by itself, and the damage was only diagnosable after application of a self-adaptive noise cancellation filter.

Machine learning based method have also seen an increasing interest for damage detection of rotating machinery due to their capabilities of classifying signals under blind assumptions [4,5]. Usually a set of features, statistical features for example, are used as inputs to train and test the model. The main disadvantage is that they need a high number of signals (samples) in order to properly train the classification model.

The last decade, the interest in the development and use of cyclostationary based tools for condition monitoring, such as the Cyclic Spectral Correlation (CSC) and the Cyclic Spectral Coherence (CSCoh), has been increased as they achieve high performance in revealing hidden periodicities in their bi-variable representations. Direct analysis of these results is difficult to perform, unless the analyst has extensive prior experience. On the other hand, the integration of the spectral frequency variable results in a one dimensional envelope spectrum, the Enhanced Envelope Spectrum (EES), detailing the cyclic modulations present in the signal. Furthermore, when the integration is performed on a specific narrow band instead of the full band, only the signature with carriers inside the integrated band are kept in the spectrum, nominated as the Improved Envelope Spectrum (IES). This method achieves robust results in extracting hidden modulations in the spectrum, and results in a classical Envelope Spectrum of easy analysis [8,9]. Recently, the IESFOgram (Improved Envelope Spectrum via Feature Optimization -gram) has been proposed as an automated band selection tool for application on the CSC and CSCoh bi-variable maps, leading to robust results in the field of bearing diagnostics [9].

The key objective of this paper is to evaluate the performance of the IESFOgram and compare it with state of the art methods by applying it on real vibration data acquired from a MGB of a Super Puma under various levels of damage on the planet bearing, operating under different speed and load conditions.

\section{Band selection tools theory}

\subsection{Fast Kurtogram}

Fast Kurtogram is a fourth-order spectral analysis tool which has been introduced for detecting and characterizing non-stationarities in a signal and it has been widely used as an automated band selection tool based on the maximization of Spectral Kurtosis (SK). Therefore a $1 / 3$ binary tree filter bank structure is prepared and the SK of each band is estimated. The band where the SK is maximized corresponds to an optimal filter band which presents a high Signal-to-Noise Ratio (SNR) leading to a Squared Envelope Spectrum (SES) 
after demodulation, where the fault harmonics are enhanced. On the other hand it has been proven that Kurtogram is sensitive to outliers.

\subsection{IESFOgram}

Rolling element bearing signals can be described as second-order cyclostationary signals, due to the slippage of elements, presenting a periodic autocorrelation of period $\mathrm{T}$ :

$$
R_{2 x}(\tau, t)=\mathbb{E}\left\{x(t) x(t-\tau)^{*}\right\}=R_{2 x}(\tau, t+T)
$$

where $\mathrm{x}(\mathrm{t})$ is the time signal, $\mathbb{E}$ is the ensemble average and $\tau$ is the time-lag. Cyclostationary based tools, such as Cyclic Spectral Correlation (CSC) and Coherence (CSCoh) have received an ever increasing interest due to their ability in extracting hidden modulations. CSC estimates the correlation between a modulating frequency (the cyclic frequency $\alpha$ ) and a carrier frequency (the spectral frequency f) presented in a bi-variable map [7]:

$$
\operatorname{CSC}(\alpha, f)=\lim _{T \rightarrow \infty} T^{-1} \mathbb{E}\left[\mathcal{F}(x(t))^{*} \mathcal{F}(x(t-\tau))\right]
$$

where $F(x(t))$ is the Fourier transform of the signal $x(t)$, and $\mathbb{E}$ is the ensemble average.

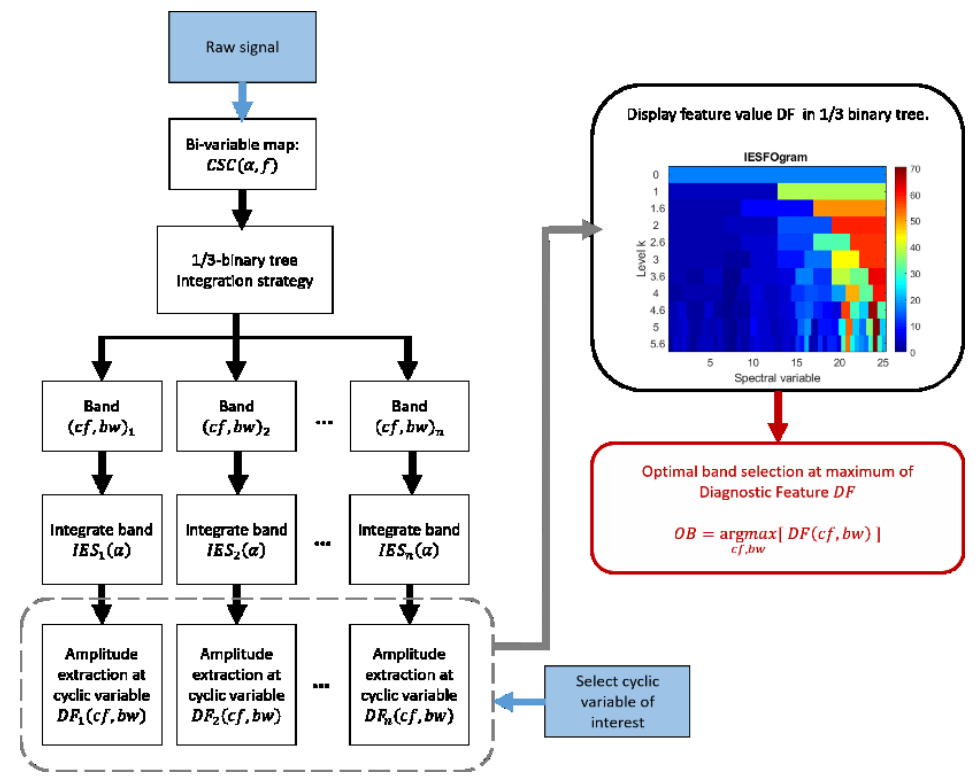

Figure. 1. Schematic description of the IESFOgram procedure. CSC:

The Cyclic Spectral Coherence (CSCoh) is the whitened version of the

$$
\operatorname{CSCoh}(\alpha, f)=\frac{\csc (\alpha, f)}{\sqrt{ } \operatorname{CsC}(0, f) \cdot \operatorname{CSC}(0, f-\alpha)}
$$

The integration of the bi-variable maps over a band $\left[\mathrm{F}_{1}, \mathrm{~F}_{2}\right]$ of the spectral frequency $f$ leads to the Improved Envelope Spectrum (IES) and to the Enhanced Envelope 
Spectrum (EES) $\left(F_{1}=0, F_{2}=F_{s} / 2, F_{s}\right.$ is the sampling frequency) which are equivalent to a demodulation spectrum:

$$
\operatorname{IES}(\alpha) \underset{F_{2}-F_{1}}{1} \int_{F_{1}}^{F_{2}}|\operatorname{CSCoh}(\alpha, f)| d f
$$

Recently the IESFOgram has been proposed as a tool is order to automatically select the optimal integration band, similarly to the Kurtogram [8]. A series of integrating iterations for different combinations of bandwidth and centre frequency are performed following a $1 / 3$ binary tree. A diagnostic feature (DF), equal to the sum of the amplitude of $k=3$ harmonics of the bearing fault frequency ffault normalized by the surrounding noise level (estimated at a band $\left.\left[f_{\text {fault }-} /+1 / 4 f_{\text {shaft }}\right]\right)$, is estimated at each band, as described by Eq. (5).

$$
D F=\sum_{k=1}^{3} \frac{I E S\left(k \times \alpha_{f a u l t}\right)}{\frac{1}{2 f_{b}}\left[\int_{k \alpha_{f a u l t}-f_{b}}^{k \alpha_{f a u l t}} f_{b} I E S(\alpha) d \alpha-I E S\left(k \times \alpha_{f a u l t}\right)\right]}
$$

The highest feature value corresponds to the optimal integration band as described in Figure 1 .

\section{Experimental Setup}

A Category A Super Puma SA330 main Gearbox (MGB) has been used in order to investigate the accuracy of planetary bearing defect diagnosis methods. The measurements have been acquires from a dedicated test rig, presented in Fig. 2, in the frames of a project entitled "MGH - Helicopter main gearbox health" sponsored by the European Aviation Safety Agency (EASA).

(a)

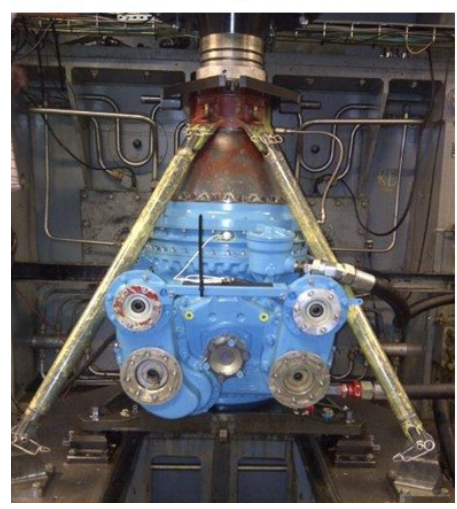

(b)

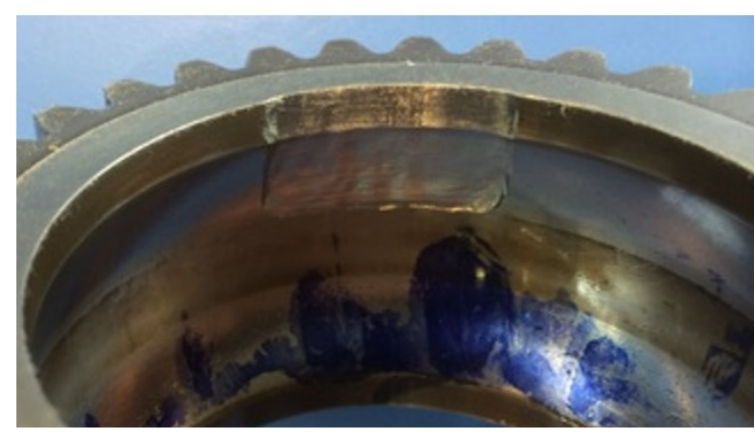

Figure. 2. (a) Tested Main Gearbox, (b) Major fault seeded on the planet outer race.

The MGB has several stages of reduction, parallel and planetary. Bearing defects have been seeded in the outer race of the planetary bearing at the second epicyclic module. More precisely, the outer race faults have been seeded on the inner bore of the planet gear, which is the outer race of the bearing. The planet gears are driven by the 2 nd epicyclic sun gear and the ring gear. 


\section{Results}

For each case, different number of accelerometers were used, but the authors show the results in this section for the accelerometer signal that is closest to the damage source and that is not corrupted or truncated.

\subsection{Case under $23,000 \mathrm{rpm}$ and $1760 \mathrm{~kW}$ operating conditions}

The vibrations signals analysed in this first case were captured under 23,000 rpm shaft speed and $1760 \mathrm{~kW}$. The signals were also measured for three cases health conditions of the outer race of the planet bearing of the second epyciclic stage: healthy, minor fault, and major fault.

The minor fault is approximately $10 \mathrm{~mm}$ wide, and the major fault is approximately $30 \mathrm{~mm}$. Based on the kinematics of the main gearbox, the frequency of interest can be calculated to be the BPFO, with a cyclic frequency of $97.4 \mathrm{~Hz}$. Detection of this component and its harmonics results in the diagnosis of the damage.

The SES and EES were also extracted and analysed, however no peaks of the BPFO were detected in any of the cases.

The Fast Kurtogram for each health condition, seen in Fig. 3, were used to select the band for filtering that results in the SES based on Fast Kurtogram seen in Fig. 4. Low kurtosis values are represented in blue, and the optimal band of each Fast Kurtogram is represented in yellow as the highest kurtosis value.
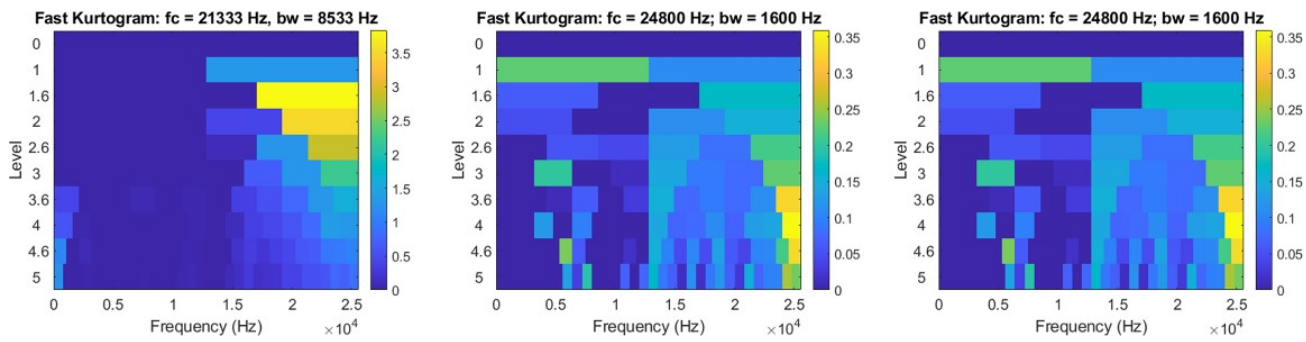

Figure. 3. Fast Kurtogram of: (left) healthy case, (middle) minor fault case, and (right) major fault case
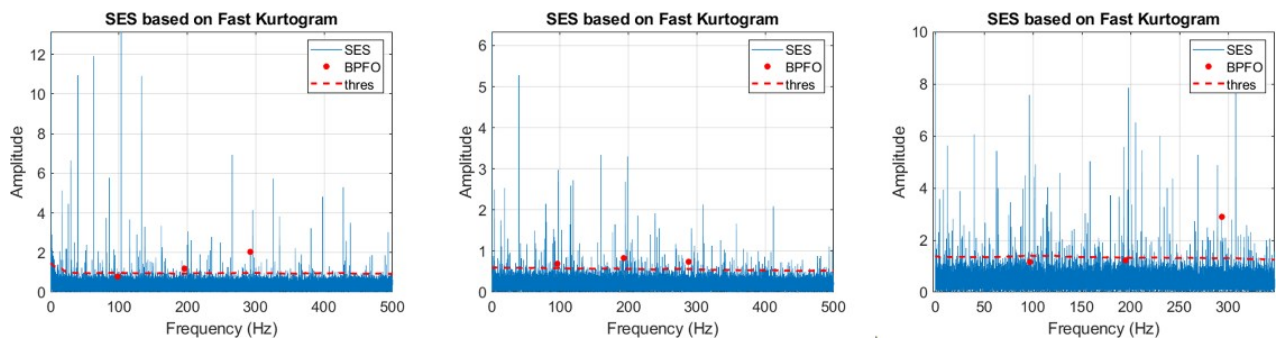

Figure. 4. Squared Envelope Spectrum based on the Fast Kurtogram of: (left) healthy case, (middle) minor fault case, and (right) major fault case.

The harmonics of the BPFO cannot be detected with confidence above the noise level, as can be seen on the spectra. 
On the other hand, the IESFOgram for the same signals, seen in Fig. 5, and its corresponding IES, seen in Fig. 6, detect the peaks at the BPFO's harmonics well above the noise level for the damaged cases, and thus providing a robust diagnosis.
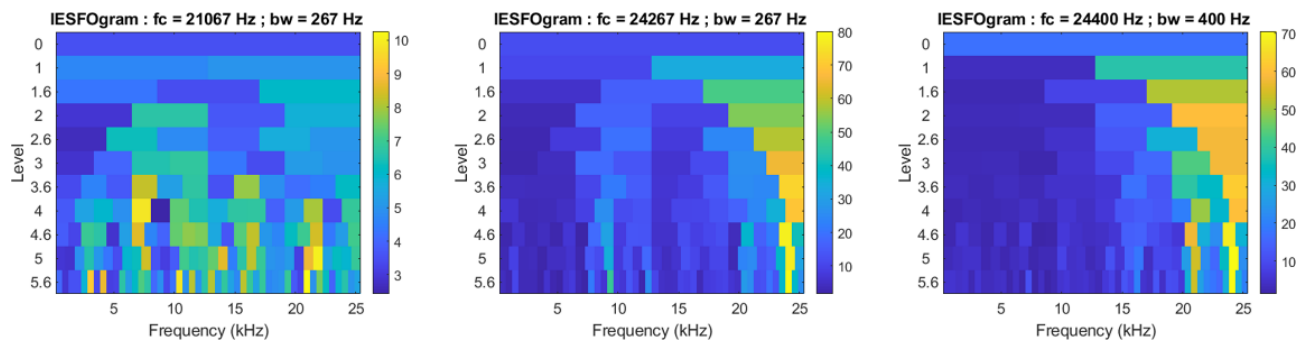

Figure. 5. IESFOgram of: (left) healthy case, (middle) minor fault case, and (right) major fault case
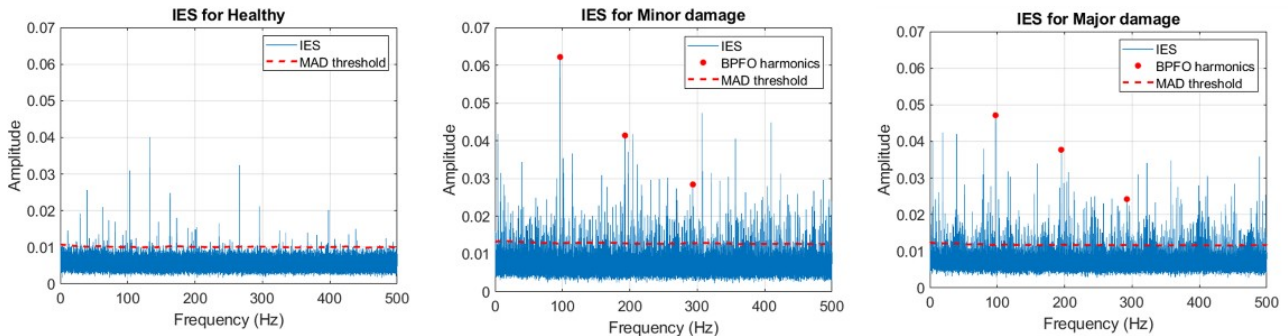

Figure. 6. Improved Envelope Spectrum based on the IESFOgram of: (left) healthy case, (middle) minor fault case, and (right) major fault case.

\subsection{Case under $16,000 \mathrm{rpm}$ and $180 \mathrm{~kW}$ operating conditions}

On the second test case, the operating conditions were lower than on the first case, at 16,000 rpm rotation speed and $180 \mathrm{~kW}$. The minor fault has a length, width and depth of $5 \mathrm{~mm}, 3 \mathrm{~mm}$, and $1.5 \mathrm{~mm}$ respectivelly. The major fault has in turn a length, width and depth of $8 \mathrm{~mm}, 6 \mathrm{~mm}$, and $2.5 \mathrm{~mm}$. Under these speed conditions, the BPFO is $68.5 \mathrm{~Hz}$.

The peaks at the BPFO harmonics were not detectable for any health condition for the SES, the EES, and the SES based on the Fast Kurtogram, as seen in Fig. 7.
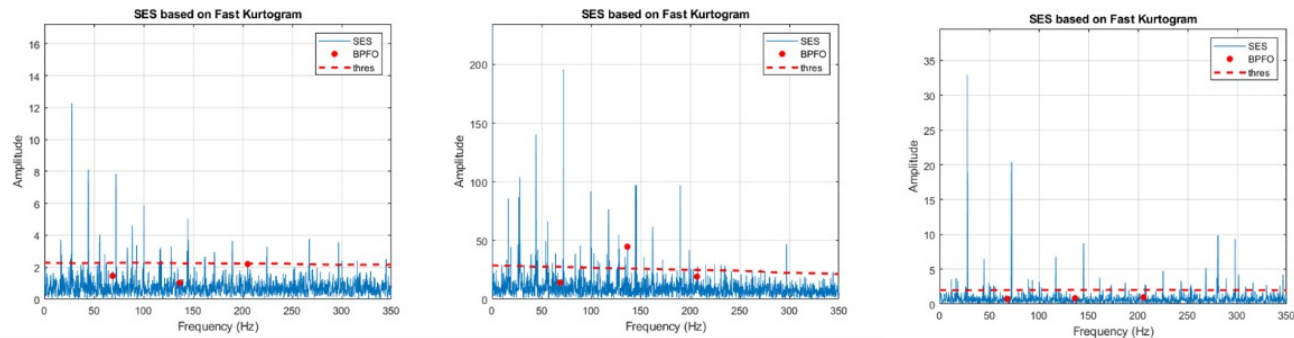

Figure. 7. Squared Envelope Spectrum based on the Fast Kurtogram of: (left) healthy case, (middle) minor fault case, and (right) major fault case.

The IES based on the IESFOgram exhibits the first harmonic of the BPFO well above the noise level, however the peak at the BPFO is also detected in the healthy case. The amplitude 
increases with the severity level, which can provide a diagnosis of increasing damage, but the detection of the BPFO at the healthy case does not provide full confidence in the diagnosis. The authors can infer that the peak at the $68.5 \mathrm{~Hz}$ in the healthy case can be due to the damage of the outer race during the bearing mounting in the setup, the ball pass frequency due to varying stiffness from the balls in contact, or other component altogether [10].
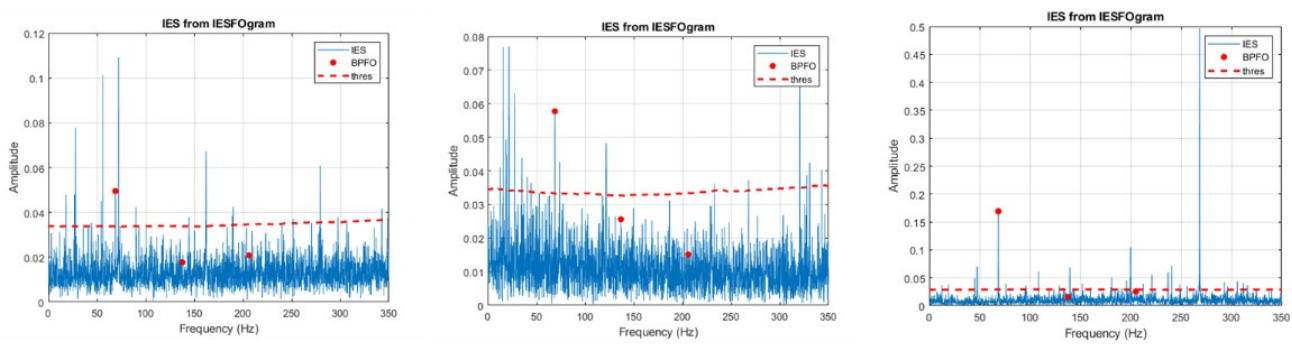

Figure. 8. Improved Envelope Spectrum based on the IESFOgram of: (left) healthy case, (middle) minor fault case, and (right) major fault case.

\subsection{Case under $23,000 \mathrm{rpm}$ and $1300 \mathrm{~kW}$ operating conditions}

The last case was only captured for two health conditions: healthy and major damage on the outer race of the bearing. The setup was under $23,000 \mathrm{rpm}$ and $1300 \mathrm{~kW}$ operating conditions on both situations.

On this case, the peaks at the $97.4 \mathrm{~Hz}$ are more easily extracted when compared to the previous cases, as the BPFO can be detected not only on the IES based on the IESFOgram, ass seen in Fig. 9, but also with the classical SES and EES. The only exception is the SES based on the Fast Kurtogram, where the BPFO harmonics are not detectable on the major fault spectrum.

(a)

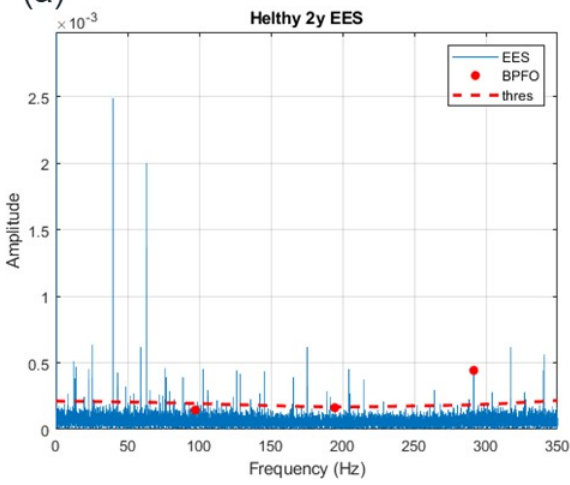

(b)

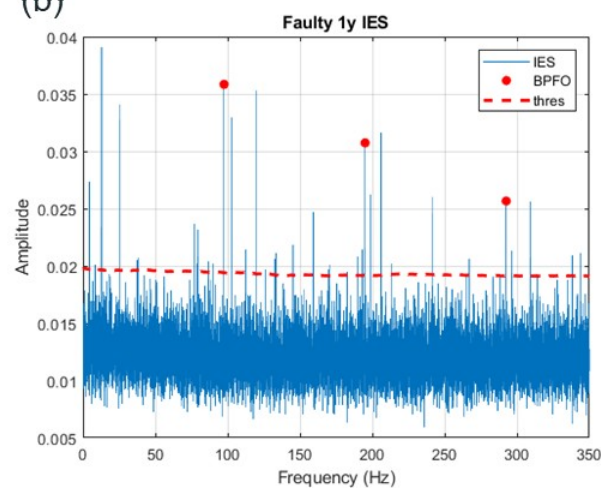

Figure. 9. Improved Envelope Spectrum based on the IESFOgram of: (left) healthy case, and (right) major fault case.

\subsection{Comparison of methodologies}

The results of the methodologies' comparison are briefly presented in Table 1 for the three analysed cases. The "NO" marks the NO fault detection while the "YES" highlights the fault detection. The green colour corresponds to the correct result while the red colour corresponds to an error. The Fast Kurtogram based SES shows low performance, either because the signal 
of interest is well masked by other components, or because an incorrect filtering band is selected. Moreover the diagnosis by the classical SES and EES analysis is successful for only one of the three cases. The integration of the CSCoh using the band selected from the IESFOgram results in spectra where the BPFO is clearly identified in all the damaged cases,

Table. 1. Detection of the BPFO for all cases for different signal processing methods.

\begin{tabular}{|c|c|c|c|c|c|}
\hline Operating conditions & Damage & SES & EES & IES & $\begin{array}{c}\text { Fast } \\
\text { Kurtogram }\end{array}$ \\
\hline \multirow{2}{*}{$\begin{array}{c}\text { Speed } 23,000 \mathrm{rpm} \\
\text { Load } 1760 \mathrm{~kW}\end{array}$} & Healthy & NO & NO & NO & NO \\
\cline { 2 - 6 } & Minor Damage & NO & NO & YES & NO \\
\cline { 2 - 6 } & Major Damage & NO & NO & YES & NO \\
\hline \multirow{2}{*}{$\begin{array}{c}\text { Speed } 23,000 \mathrm{rpm} \\
\text { Load } 1760 \mathrm{~kW}\end{array}$} & Healthy & NO & NO & YES & NO \\
\cline { 2 - 6 } & Minor Damage & NO & NO & YES & NO \\
\cline { 2 - 6 } & Major Damage & NO & NO & YES & NO \\
\hline $\begin{array}{c}\text { Speed } 23,000 \mathrm{rpm} \\
\text { Load } 1760 \mathrm{~kW}\end{array}$ & Healthy & NO & NO & NO & NO \\
\cline { 2 - 6 } & Major Damage & YES & YES & YES & NO \\
\hline
\end{tabular}

however it is also extracted on the healthy signal of one of the cases. Based on Table 1 it can be concluded that the IES estimated based on the IESFOgram achieves the best results compared to the other 3 state of the art methodologies. On the other hand, one false alarm is present and the authors are now working on this issue in order to clarify the reason of appearance and the improvement of the general methodology targeting towards a robust diagnostic method with a limited rate of false alarms and missed detections.

\section{Conclusions}

In this paper, the IES based on the IESFOgram is proposed for diagnosis of bearing diagnostics of helicopter's main gearbox. The procedure is applied over signals captured on a Category A Super Puma SA330 helicopter main gearbox transmission, for various speed conditions, load conditions and severity levels of damage on a rolling element bearing. The procedure is compared against the classical SES, the EES and the SES based on the Fast Kurtogram. The detection of the BPFO for all cases and the mentioned methods are summarized in Table 1.

The Fast Kurtogram based SES shows low performance, either because the signal of interest is well masked by other components, or by selecting the incorrect band for filtering. Diagnosis by the classical SES and EES analysis is robust for one of the three cases. The integration of the CSCoh using the band selected from the IESFOgram results in spectra where BPFO are clearly identified in all the damaged cases, however the BPFO it is also extracted on the healthy signal of one of the cases. Therefore early detection of the faults can be extracted with the use of IESFOgram.

\section{References}

1. L. Zhou, F. Duan, M. Corsar, F. Elasha, D. Mba, Applied Acoustics. 147, 4-14 (2019)

2. R. Randall, N. Sawalhi, Rot. Machinery, Struct. Health Moni,, Shock and Vibr., 5, 451- 461 (2011) 
3. W. A. Smith, Z. Fan, Z. Peng, H. Li, R. Randall, MSSP, 75, 371-394 (2016)

4. Z. Chen, A. Mauricio, W. Li, K. Gryllias, MSSP, 140, 106683 (2020)

5. L. Wen, X. Li, L. Gao, Y. Zhang ,IEEE Trans. Ind. Electron., 65, 5990-5998 (2018),

6. R. Randall, J. Antoni, J. Chobsaard, MSSP, 15, 945-962 (2001)

7. D. Abboud, S. Baudin, J. Antoni, D. Remond, M. Eltabach, O, Sauvage, MSSP, 75, 280- 300 (2016)

8. A. Mauricio, J. Qi, K. Gryllias, Proceedings of ASME TurboExpo (2018)

9. A. Mauricio, W. Smith, R. Randall, J. Antoni, K. Gryllias, Proc. ISMA, 17-19 Sep. (2018)

10. H. Cheng, Y. Zhang, W. Lu, Z. Yang, Measurement, 143, 155-179 (2019) 